\title{
Acid-Catalyzed Hydrolysis of Bridged Bi- and Tricyclic Compounds. XXVI. The Mechanisms of Formation of the Hydration Products of 2-Norbornen-5-one
}

\author{
Martti Lajunen, Marjo Lahti and Satu Heimo \\ Department of Chemistry and Biochemistry, University of Turku, SF-20500 Turku 50, Finland
}

\begin{abstract}
Lajunen, M., Lahti, M. and Heimo, S., 1989. Acid-Catalyzed Hydrolysis of Bridged Bi- and Tricyclic Compounds. XXVI. The Mechanisms of Formation of the Hydration Products of 2-Norbornen-5-one. - Acta Chem. Scand. 43: 771-776.

The acid-catalyzed hydration of 2-norbornen-5-one (1) in $5 \mathrm{~mol} \mathrm{dm}^{-3} \mathrm{HClO}_{4}$ at $358 \mathrm{~K}$ produces, according to GLC analyses, exo-5-hydroxy-2-norbornanone (2), 2-oxabicyclo[3.2.1] octan-3-one (3), which is in equilibrium with cis-3-hydroxycyclopentaneacetic acid (5), and 2-oxabicyclo[3.3.0]octan-3-one (4). The former lactone (3) is unstable and rearranges slowly to the latter (4). The ketoalcohol (2) is formed via the slow protonation of norbornenone at $\mathrm{C}(3)$ and via subsequent hydration of the carbocation. The lactones are formed via complicated routes started by the protonation of norbornenone at $\mathrm{C}(2)$, and comprising 3- and 2-cyclopenteneacetic acids (7 and 9) as unstable intermediate products.
\end{abstract}

The hydration of 2-norbornen-5-one (a special $\beta, \gamma$-unsaturated ketone) in aqueous acid was investigated kinetically about ten years ago. ${ }^{1}$ The protonation of the carbon-carbon double bond was concluded to be the rate-limiting stage of the reaction on the basis of the activation entropy, solvent deuterium isotope effect and the effects of methyl substitution. In accordance with the product analysis made by Krieger $^{2}$ of the sulfuric acid catalyzed addition of acetic acid to norbornenone, the hydration products were supposed (although they were not analyzed) to be exo-5- and exo-6-hydroxy-2-norbornanones (eqn. 1). The amount of

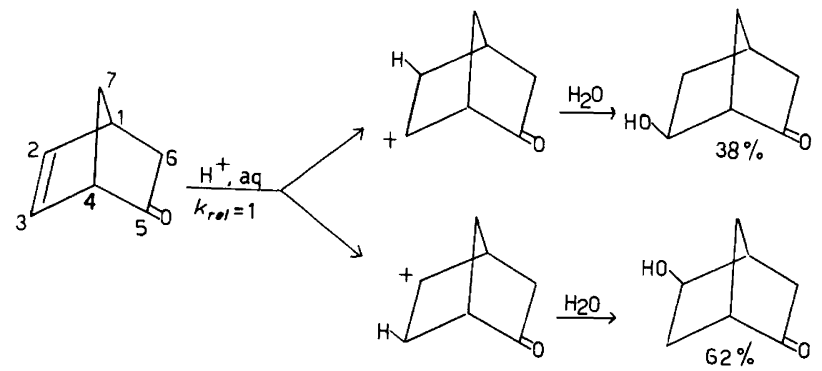

the former ketoalcohol was estimated to be somewhat greater, because a methyl substituent at $\mathrm{C}(2)$ of norbornenone accelerates the protonation of the double bond more than a methyl substituent at $\mathrm{C}(3)$ (eqns. 2 and 3). ${ }^{1}$<smiles>CC1=CC2CC1CC2[C@@H]1C[C@H]2C[C@@H](C1)C2O</smiles>

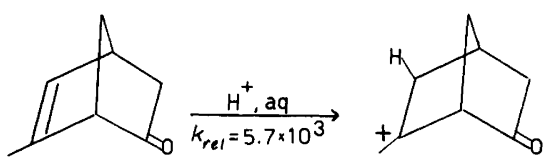

(3)

Carrupt and Vogel $^{3}$ recently studied the products of electrophilic additions of benzeneselenyl- and arenesulfenyl halides to norbornenone (eqn. 4) and 5-chloro-5-cyano-2norbornenes (eqn. 5) in different solvents. Their results are in agreement with ours ${ }^{4}$ in the latter case, since the electrophile $\left(\mathrm{PhSe}^{+}\right.$) used by them attacks solely $\mathrm{C}(3)$ (eqn. 5) of 5-cyano-2-norbornenes, and that $\left(\mathrm{H}^{+}\right)$used by us attacks mostly C(3) (eqn. 6). However, their results differ from

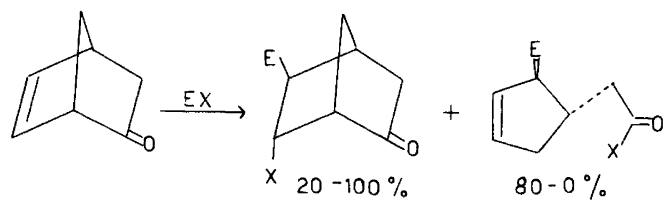

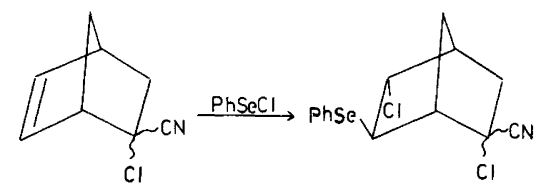

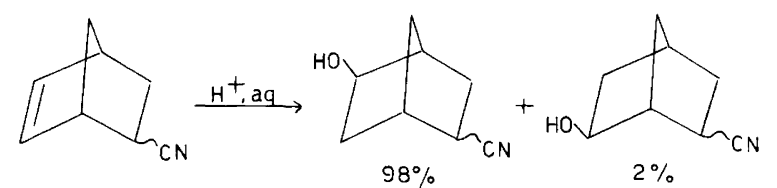


ours $^{1}$ in the case of norbornenone, since the electrophiles $\left(\mathrm{PhSe}^{+}\right.$and $\left.\mathrm{ArS}^{+},=\mathrm{E}\right)$ used by them attack exclusively $\mathrm{C}(2)$ (eqn. 4), but that $\left(\mathrm{H}^{+}\right)$used by us attacks $\mathrm{C}(2)$ and $\mathrm{C}(3)$ in the ratio 38:62 according to our kinetic data (eqns. 2 and 3). Carrupt and Vogel $^{3}$ also observed that a "hard" electrophile, especially in solvents of strong ionizing power, causes fragmentation of the intermediate cation and formation of monocyclic products (eqn. 4). This kind of fragmentation has also been found in the solvolyses of 2-norbornyl tosylates containing a strongly electron-donating substituent (e.q. $\mathrm{OH}$ or $\mathrm{OCH}_{3}$ ) at $\mathrm{C}(6)$, and it has been observed to cause a marked increase of reaction rate (a so-called frangomeric effect). ${ }^{5}$

The proton is a hard electrophile and water is a solvent of strong ionizing power. Thus, the fragmentation might also dominate in the hydration of norbornenone, since the oxo group is evidently also such an electron-donating (frangomeric) substituent which enforces a possible positive charge formation initially at $C(3)$, wherefrom it transfers to the carbonyl group. ${ }^{1.6}$ The attack (or shift) of a proton at $\mathrm{C}(2)$ is, however, a condition for the fragmentation in the acidcatalyzed hydration. In order to see whether the hydration products of norbornenone are different from those supposed earlier and/or whether they are in conflict with the ratio of protonation at $C(2)$ and $C(3)$ obtained by a kinetic methyl-substituent method,' the products of acid-catalyzed hydration of 2-norbornen-5-one(1) were studied and their routes of formation are discussed in the present paper.

\section{Experimental}

Syntheses. 2-Norbornen-5-one (1) was prepared by hydrolyzing the Diels-Alder adduct of cyclopentadiene and 2chloroacrylonitrile in alkaline DMSO. ${ }^{7}$ Its purity was $98.6 \%$, and ${ }^{1} \mathrm{H}$ and ${ }^{13} \mathrm{C}$ NMR spectra were identical with the reported ones. ${ }^{8.9}$

exo-5-Hydroxy-2-norbornanone (2) was prepared by addition of acetic acid to 3-nortricyclanone, catalyzed by sulfuric acid, and hydrolysis of the product, exo-5-acetoxy-2norbornanone, in aqueous $\mathrm{KOH} .^{2}$ Ketoalcohol 2 is very soluble in aqueous media, and repeated ether extraction of the solution was therefore carried out. Yield $58 \%$; purity $99 \% ;{ }^{13} \mathrm{C}$ NMR $\left(15.0 \mathrm{MHz}, \mathrm{CDCl}_{3}\right): \delta 49.0(\mathrm{C}-1), 217.5$ (C-2), 40.1 (C-3), 43.2 (C-4), 72.2 (C-5), 35.5 (C-6) and $33.6(\mathrm{C}-7)$. The IR spectrum was similar to that reported. ${ }^{2}$

2-Oxabicyclo[3.2.1]octan-3-one (lactone of cis-3-hydroxycyclopentaneacetic acid) (3) was obtained by oxidizing 2norbornanone with 3-chloroperbenzoic acid in $\mathrm{CH}_{2} \mathrm{Cl}_{2}$ in the presence of $p$-toluenesulfonic acid at room temperature for 2-3 d. ${ }^{10}$ Yield $75 \%$; purity $96 \% ;{ }^{13} \mathrm{C}$ NMR $(15.0 \mathrm{MHz}$, $\left.\mathrm{CDCl}_{3}\right): \delta 81.1(\mathrm{C}-1), 170.8(\mathrm{C}-3), 29.3,31.9,32.5,35.8$ and 40.7 (other carbon atoms); MS [IP $70 \mathrm{eV} ; \mathrm{m} / \mathrm{e}$ (\% rel. int.)]: 126 (11, M), 98 (21, [M-CO]), 97, [M-CHO]), 83 (70, $\left.\left[\mathrm{M}-\mathrm{CH}_{3} \mathrm{CO}\right]\right), 82\left(100,\left[\mathrm{M}-\mathrm{CO}_{2}\right]\right), 70(25), 69$ (48), 68
(12), 67 (94, [M- $\left.\left.\mathrm{CO}_{2} \mathrm{CH}_{3}\right]\right), 56$ (19), 55 (61), 54 (57) etc. The IR spectrum was in agreement with that reported. ${ }^{11}$

2-Oxabicyclo[3.3.0/octan-3-one (lactone of cis-2-hydroxycyclopentaneacetic acid) (4) was prepared by stirring (a) 2-norbornenone, (b) lactone (3) or (c) 2-cyclopenteneacetic acid in $5 \mathrm{~mol} \mathrm{dm}^{-3} \mathrm{HClO}_{4}$ at ca. $358 \mathrm{~K}$ for ca. $66 \mathrm{~h}$ and extracting the products with ether or $\mathrm{CH}_{2} \mathrm{Cl}_{2}$. In case (a), the ether solution was washed with water until ketoalcohol 2 was totally removed. The organic solution was dried over $\mathrm{Na}_{2} \mathrm{SO}_{4}$, the solvent was evaporated and the product was distilled in vacuo (b.p. $385.7 \mathrm{~K} / 0.48 \mathrm{kPa}$ ). Yield 10-32\%; purity $95-97 \% ;{ }^{13} \mathrm{C}$ NMR $\left(15.0 \mathrm{MHz}, \mathrm{CDCl}_{3}\right): \delta 86.5$ (C-1), 177.8 (C-3), 23.5, 33.5, 33.6, 36.1 and 38.0 (other carbon atoms). The IR, ${ }^{1} \mathrm{H}$ NMR and MS spectra were in agreement with those reported. ${ }^{12-14}$

2-Cyclopenteneacetic acid (9) was a commercial product from Merck-Schuchardt (purity $98 \%$ ). ${ }^{13} \mathrm{C}$ NMR (15.0 $\mathrm{MHz}, \mathrm{CDCl}_{3}$ ): $\delta 31.9$ (C-1), 133.5 (C-2), 131.7 (C-3), 40.3 (C-4), 29.7 (C-5), 41.9 (C-6) and 179.6 (C-7) (these assignments are tentative).

A mixture of 2- and 3-cyclopenteneacetic acids (9 and 7) was obtained by hydrolyzing 2-norbornenone in moist DMSO in the presence of tert-BuOK. ${ }^{15}$ Yield $53 \%$; purity $95 \%$ (containing $4 \%$ of tert-BuOH). The mixture of the acids gave only a single GLC peak, but contained $46 \%$ of $2-$, and $54 \%$ of 3-cyclopenteneacetic acids according to NMR analyses. ${ }^{13} \mathrm{C}$-NMR for the 3 -isomer $\left(15.0 \mathrm{MHz}, \mathrm{CDCl}_{3}\right): \delta 33.6$ (C-1), 38.8 (C-2), 129.5 (C-3), 40.5 (C-6) and 179.4 (C-7) (these assignments are tentative). The IR and ${ }^{1} \mathrm{H}$ NMR spectra of the components were in agreement with those reported. ${ }^{15.16}$

Product analyses. The disappearance of 2-norbornenone and its probable intermediate products, and the formation of hydrolysis products in $5 \mathrm{~mol} \mathrm{dm}^{-3}$ aqueous $\mathrm{HClO}_{4}$, usually at $358 \mathrm{~K}$, were followed by GLC by taking samples after appropriate intervals and either [method (a)] by neutralizing them with concentrated ammonia, centrifuging to isolate the precipitated salt, and analyzing the aqueous solutions by gas chromatography, generally with an FFAP packed column (the ketoalcohol was always analyzed in this way), or [ method (b)] by extracting the samples with $\mathrm{CH}_{2} \mathrm{Cl}_{2}$ and analyzing the organic solutions by gas chromatography with an XE-60 or NB-225 capillary column. Nitrobenzene, or $o$ - or $m$-nitrotoluene were used as internal standards. The analyses of ketoalcohol 2 and lactone 3 were not accurate, possibly due to their decomposition, especially when analyzing the aqueous solutions. Each run and analysis was repeated at least once.

The relative peak areas for the components were corrected according to their response ratios $(R R)$ determined separately in $\mathrm{CH}_{2} \mathrm{Cl}_{2}$ or acetone and in water (eqn. 7). In

$$
R R_{\mathrm{i}}=\left(a_{\mathrm{i}} / n_{\mathrm{i}}\right) /\left(a_{1} / n_{1}\right)
$$


Table 1. Disappearance rate constants for 2-norbornen-5-one and for some for its hydration products in $5.0 \mathrm{~mol} \mathrm{dm}^{-3} \mathrm{HClO}_{4}$, and activation parameters at $348.2 \mathrm{~K}$.

\begin{tabular}{lccc}
\hline Substrate & $T / \mathrm{K}$ & $k_{1} / 10^{-4} \mathrm{~s}^{-1}$ & Activation parameters \\
\hline 2-Norbornen-5-one & 358.2 & $1.22(10)^{a}$ & $\Delta H^{+}=109(2) \mathrm{kJ} \mathrm{mol}^{-1 b}$ \\
& & & $\Delta S^{\neq}=-30(6) \mathrm{J} \mathrm{mol}^{-1} \mathrm{~K}^{-1 b}$ \\
2-Oxabicyclo[3.2.1]octan-3-one & 358.2 & $0.44(4)^{c}$ & \\
Mixture of 2- and 3-cyclopenteneacetic acids & 338.2 & $4.07(1)$ & \\
& 348.2 & $12.9(1)$ & \\
2-Cyclopenteneacetic acid & 358.2 & $33.3(6)$ & $\Delta H^{+}=104(6) \mathrm{kJ} \mathrm{mol}^{-1}$ \\
& 338.2 & $3.42(14)$ & $\Delta S^{+}=-17(18) \mathrm{J} \mathrm{mol}^{-1} \mathrm{~K}^{-1}$ \\
3-Cyclopenteneacetic acid & 348.2 & $11.3(7)$ & $\Delta H^{+}=100(6) \mathrm{kJ} \mathrm{mol}^{-1}$ \\
& 358.2 & $28.3(9)$ & $\Delta S^{+}=-27(18) \mathrm{J} \mathrm{mol}^{-1} \mathrm{~K}^{-1}$
\end{tabular}

${ }^{a} \mathrm{~A}$ mean value of the measurements by methods (a) and (b). ${ }^{b}$ Ref. 1. ${ }^{c}$ The rate constant measured for the formation of 2-oxabicyclo[3.3.0]octan-3-one is $(3.7 \pm 0.4) \times 10^{-5} \mathrm{~s}^{-1}$. ${ }^{d}$ The ratio of the isomers is $46: 54$, respectively.

this equation, $a_{\mathrm{i}}=$ integral of component $\mathrm{i}$ in a chromatogram, $n_{\mathrm{i}}=$ (mass of $\left.\mathrm{i}\right) \mathrm{x}($ GLC purity of $\mathrm{i}) /($ molecular weight of i). The subscript 1 refers to 2-norbornenone. The response ratios of ketoalcohol (2) and lactones (3) and (4) were markedly lower in water than in organic solvents.

Isomerization of 2-cyclopenteneacetic acid (9) to 3-cyclopenteneacetic acid (7) was studied by stirring a solution (50 $\mathrm{cm}^{3}$ ) of the former acid $\left(0.5 \mathrm{~cm}^{3}\right)$ in $5 \mathrm{~mol} \mathrm{dm} \mathrm{dm}^{-3} \mathrm{HClO}_{4}$ for 20 or 40 min at $343 \mathrm{~K}$, extracting the solution with $\mathrm{CH}_{2} \mathrm{Cl}_{2}$, drying the organic solution over $\mathrm{Na}_{2} \mathrm{SO}_{4}$, evaporating the solvent in vacuo and analyzing the residue by NMR $\left({ }^{1} \mathrm{H}\right.$ and ${ }^{13} \mathrm{C}$ ) in $\mathrm{CDCl}_{3}$. The amount of the 3 -isomer could be estimated from the integrals of the signals for the olefinic hydrogens. ${ }^{16}$

Equilibria of lactones (3 and 4) with hydroxycyclopentaneacetic acids ( 5 and $\mathbf{6}$ ) were studied by alkali titration of the hydroxyacids formed from the lactones (initially $0.014 \mathrm{~mol}$ $\mathrm{dm}^{-3}$ ) in 0.02 and $0.1 \mathrm{~mol} \mathrm{dm}^{-3} \mathrm{HClO}_{4}$ at $298.2 \mathrm{~K}$ after 5 and 2 days, respectively. ${ }^{17}$ The low solubilities of the lactones made the measurements rather inaccurate.

\section{Results and discussion}

The disappearance rate constant (Table 1) measured in this work for 2-norbornen-5-one (1) in $5.0 \mathrm{~mol} \mathrm{dm}^{-3} \mathrm{HClO}_{4}$ at $358.2 \mathrm{~K}$ is in agreement with that measured earlier $\left(1.24 \times 10^{-4} \mathrm{~s}^{-1}\right) .{ }^{1}$ Norbornenone yields the following products detectable by GLC: exo-5-hydroxy-2-norbornanone (ketoalcohol, 2), 2-oxabicyclo[3.2.1] octan-3-one (the lactone of cis-3-hydroxycyclopentaneacetic acid) ( $\delta$-lactone, 3) and 2-oxabicyclo[3.3.0]octan-3-one (the lactone of cis-2hydroxycyclopentaneacetic acid) $(\gamma$-lactone, 4) (see Fig. 1 and Scheme 1). The ketoalcohol and the $\gamma$-lactone are practically stable products in the reaction medium, but the $\delta$-lactone rearranges to the $\gamma$-lactone at a rate which is ca. $36 \%$ of the rate of disappearance of norbornenone, (Table 1). ${ }^{18}$

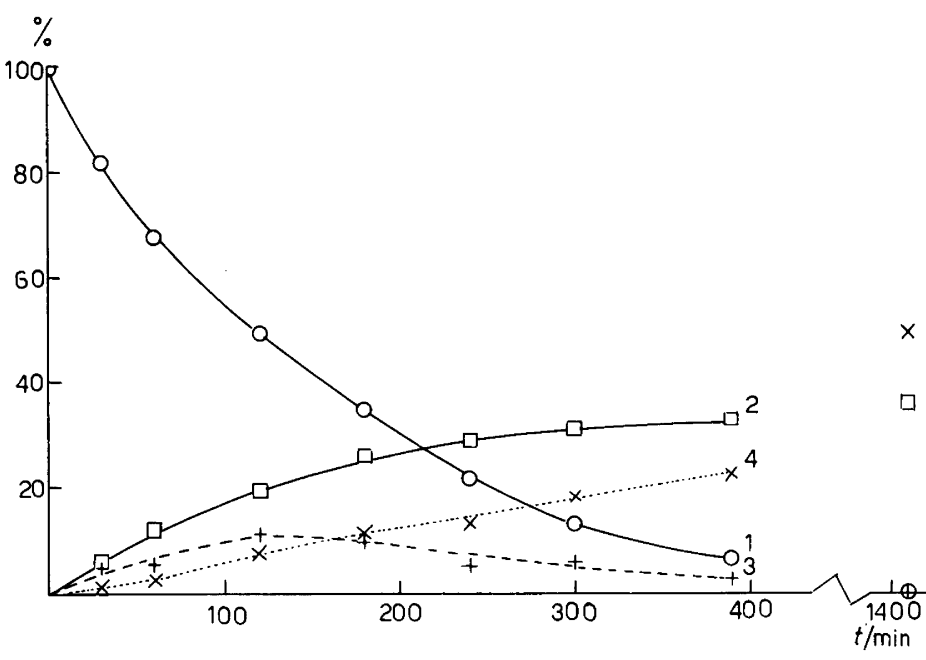

Fig. 1. The amounts ( $\mathrm{mol} \%$ ) of the substrate and products (visible in the GLC analyses) during the hydration of 2norbornen-5-one in $5.0 \mathrm{~mol} \mathrm{dm}^{-3} \mathrm{HClO}_{4}$ at $358.2 \mathrm{~K}$. Symbols: $O, 2$-norbornen-5-one (1); $\square$, exo-5-hydroxynorbornan-2-one (2); +, 2-oxabicyclo[3.2.1]octan-3-one (3); and $\times, 2$ oxabicyclo[3.3.0]-octan-3-one (4). 


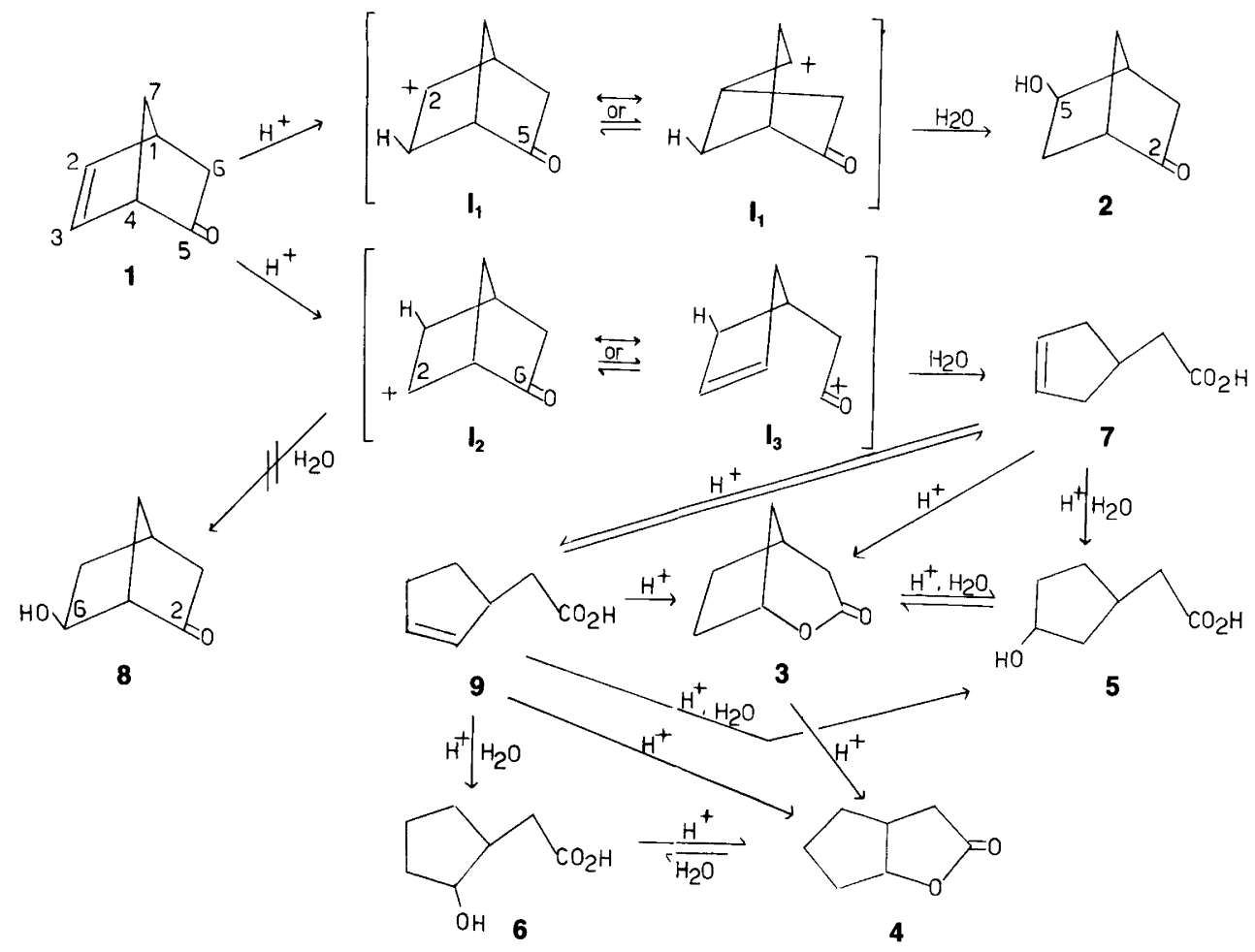

Scheme 1

The rearrangement of the $\delta$-lactone was investigated separately under the conditions mentioned above. Its disappearance followed satisfactorily, and the formation of the $\gamma$-lactone followed nicely, first-order kinetics. The GLC peak area for the $\gamma$-lactone in the final samples (taken after ten half-lives) was on an average $2.34 \pm 0.06$ times greater than that for the $\delta$-lactone in the initial samples. An evident explanation is the existence of an equilibrium between the $\delta$-lactone and cis-3-hydroxycyclopentaneacetic acid (5), with an equilibrium constant ([hydroxyacid]/[lactone]) of $1.34 \pm 0.05$. The equilibrium constant was also measured under milder conditions, viz. in 0.02 and $0.1 \mathrm{~mol} \mathrm{dm}^{-3}$ $\mathrm{HClO}_{4}$ at $298.2 \mathrm{~K}$, and the resulting values $(0.81 \pm 0.07$ and $1.26 \pm 0.12$, respectively) agree fairly well with the value above. Thus, the acid concentration and temperature have no marked effect on the equilibrium constant.

An equilibrium between the $\gamma$-lactone and cis-2-hydroxycyclopentaneacetic acid (6) was also studied in 0.02 and 0.1 mol $\mathrm{dm}^{-3} \mathrm{HClO}_{4}$ at $298.2 \mathrm{~K}$. The equilibrium constants, $0.013 \pm 0.015$ and $0.038 \pm 0.035$, respectively, show that the proportion of hydroxyacid at equilibrium is very small in this case. The equilibrium constants for the $\gamma$ - and $\delta$ lactones are in agreement with those measured for substituted $\gamma$-butyrolactones and $\delta$-valerolactones. ${ }^{17}$

The formation of ketoalcohol (2), which obeys fairly well first-order kinetics, is easy to rationalize (Scheme 1) on the basis of the earlier mechanistic results. ${ }^{1}$ The carbon-carbon double bond of norbornenone (1) is slowly protonated at $\mathrm{C}(3)$, and the resulting 5-oxo-2-norbornyl cation $\left(\mathbf{I}_{1}\right)$ is subsequently attacked by a water molecule from the exo side. A possible Wagner-Meerwein rearrangement of the cation is degenerative and produces the enantiomeric ketoalcohol.

The rationalization of the formation of the lactones $(3$ and 4: Scheme 1) is more complicated. Since their amounts are considerable (Fig. 1), they are probably also formed via protonation of the carbon-carbon double bond, although in this case at $\mathrm{C}(2)$. The carbonyl group of the 6-oxo-2norbornyl cation $\left(\mathbf{I}_{2}\right)$ formed is in a proper position for donating conjugatively electrons to the carbocation center, causing fragmentation of the cation. ${ }^{3,6}$ The new 3-cyclopentenylethanyl cation $\left(\mathbf{I}_{\mathbf{3}}\right)$, which is probably more stable than the 6-oxo-2-norbornyl cation, ${ }^{6}$ is subsequently attacked by a water molecule producing 3-cyclopenteneacetic acid ( $\Delta^{3}$ acid, 7). No formation of another possible hydration product, viz. exo-6-hydroxy-2-norbornanone (8), was detected. This ketoalcohol is probably rather unstable, since an attempt to prepare it by hydrolysis of the corresponding acetate $^{19}$ in aqueous alkali failed.

The reaction of cyclopenteneacetic acid was investigated separately in the same medium. The disappearance of the mixture of 3- and 2-cyclopenteneacetic acids ( $\Delta^{3}$ - and $\Delta^{2}$ acids, 7 and 9 , in the ratio 54:46) obeyed nicely first-order kinetics, but the fit to first-order kinetics for the pure $\Delta^{2}$-acid was only satisfactory (Table 1 ). This was assumed to be due to the close similarity of the rate constants for the acids and to a possible isomerization of the acids to each other, the equilibrium constant being close to unity. The isomerization of the $\Delta^{2}$-acid to the $\Delta^{3}$-acid could also be detected by ${ }^{1} \mathrm{H}$ and ${ }^{13} \mathrm{C}$ NMR measurements (see Experi- 
mental). After $32 \%$ and $50 \%$ disappearance of the $\Delta^{2}$ isomer, $3.5 \%$ and $6 \%$ of the $\Delta^{3}$-isomer, respectively, were formed. By subtracting the calculated peak area for the $\Delta^{2}$-acid from that for the mixture in every GLC sample, the disappearance rate constant for the $\Delta^{3}$-acid could be evaluated (Table 1 ). It is $25 \%$ greater than that for the $\Delta^{2}$ iomer, and 29 times greater than the disappearance rate constant for norbornenone in $5.0 \mathrm{~mol} \mathrm{dm}^{-3} \mathrm{HClO}_{4}$ at 358.2 $\mathrm{K}$. Thus, the rate of isomerization of the $\Delta^{2}$ - and $\Delta^{3}$-acids to each other is ca. 3 times greater than the rate of hydration of norbornenone.

The disappearance rate constants for cyclopenteneacetic acids were also measured at different temperatures and the activation parameters were calculated from the data. The values are slightly disturbed by the mutual isomerization of the acids. The activation entropies, viz. -17 and $-27 \mathrm{~J}$ $\mathrm{mol}^{-1} \mathrm{~K}^{-1}$ for the $\Delta^{2}$ - and $\Delta^{3}$-isomers, respectively, are similar to the value measured for the hydration of cyclopentene $\left(-27 \mathrm{~J} \mathrm{~mol}^{-1} \mathrm{~K}^{-1}\right.$ at $\left.348.2 \mathrm{~K}\right)$, and the rate constants are $8\left(\Delta^{2}\right.$-acid $)$ and $6\left(\Delta^{3}\right.$-acid $)$ times smaller than that measured for hydration of cyclopentene. ${ }^{20}$ The data are in accordance with slow protonation of the carboncarbon double bond, since the inductive effects of the 3and 4-carboxymethyl substituents ${ }^{21}$ retard the hydration of cyclopentene.

The protonation of the carbon-carbon double bond of cyclopenteneacetic acids produces carbocations, which are subsequently attacked by present nucleophiles. The attack of a water molecule produces 3- and 2-hydroxycyclopentaneacetic acids ( 5 and 6 ; both cis- and trans-forms are possible), and the intramolecular attack of the carboxyl group produces $\delta$ - and $\gamma$-lactones ( 3 and 4 ; Scheme 1 ). The prepared mixture of cyclopenteneacetic acids produced the $\delta$ and $\gamma$-lactones in a rather steady ratio of $63: 37$, and the pure $\Delta^{2}$-acid in the ratio 45:55. Thus, a ratio of 79:21 can be calculated for the $\Delta^{3}$-acid. This is equal to that $(78: 22)$ measured for the hydration of norbornenone $30 \mathrm{~min}$ after the start of the reaction. This supports the view that 3cyclopenteneacetic acid (7) is the first uncharged intermediate product in the route started by the protonation of norbornenone at $\mathrm{C}(2)$ (see above). In the subsequent samples from the hydration of norbornenone, the proportion of the $\gamma$-lactone increased and that of the $\delta$-lactone began to decrease (Fig. 1). The rather fast isomerization of the $\Delta^{3}$ acid to the $\Delta^{2}$-isomer and the ca. ten times slower rearrangement of the $\delta$-lactone to the $\gamma$-lactone explain the variation of the ratio of the lactones with time.

The unsaturated acids (7 and 9) are not visible in the GLC analyses of the samples taken during hydration of norbornenone due to their low concentrations: they are formed slowly but disappear rapidly. It is interesting to note that the salts of the same acids are also formed as final products in the basic hydrolysis of norbornenone. ${ }^{15}$ The hydroxycyclopentaneacetic acids (5 and 6) are also undetectable by the GLC analysis technique used, because they are neutralized to their salts when the reaction is stopped by addition of ammonia [method (a); see Experi- mental] or because their solubilities in $\mathrm{CH}_{2} \mathrm{Cl}_{2}$ are poor [method (b); cf., only a trace, ca. $1 \%$, of the ketoalcohol 2 was observed by method (b) for the same reason].

It is possible to estimate roughly the total amount of the non-visible products by subtraction of the percentages of norbornenone and the visible products from one hundred (Fig. 1). The difference increases from $6 \%(30 \mathrm{~min})$ to $35 \%$ (390 min), although not steadily. A part (6 to $13 \%$ ) of the difference is evidently due to cis-3-hydroxycyclopentaneacetic acid (5), which is in equilibrium with the $\delta$-lactone. The remainder of the difference is possibly due to the trans forms of the hydroxyacids (5 and 6). The difference for the final sample $(14 \%)$ is smaller than for the previous ones. This is reasonable, since the cis form hydroxyacid 5 also isomerizes to the $\gamma$-lactone, the corresponding acid (6) of which does not occur to any appreciable extent in the equilibrium (see above).

The data above show that cyclopenteneacetic acids (7 and 9), lactones (3 and 4) and hydroxycyclopentaneacetic acids (5 and 6) are generated instead of exo-6-hydroxy-2norbornanone (8) from norbornenone. The formation of these fragmentation products supports the strong hyperconjugative effect of the carbonyl group. ${ }^{3,6}$ The same frangomeric effect also controls the ratio of protonation at $\mathrm{C}(2)$ and $\mathrm{C}(3)$ of norbornenone, since the protonation should occur almost exclusively at $\mathrm{C}(3)$ on the basis of the electron-attractive inductive effect of the carbonyl group. ${ }^{22}$ The frangomeric effect is, however, not so great as could be presumed from the product analysis made by Carrupt and Vogel (eqn. 4). ${ }^{3}$ This is probably due to the different stages of the reactions which control the formation of the products. In the case of the acid-catalyzed hydration of norbornenone, the slow protonation of the olefinic carbon atoms also determines the ratio of the formation of the ketoalcohol and the fragmentation products, but in the case of the addition of benzeneselenyl- and arenesulfenyl halides to norbornenone, the formation of the different products is determined by the attack of nucleophiles $\left(\mathrm{Cl}^{-}\right.$or $\mathrm{Br}^{-}$) on the cyclic cation $\left(\mathbf{I}_{4}\right)$.

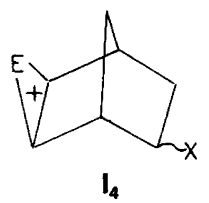

The ratio of protonation of $\mathrm{C}(2)$ and $\mathrm{C}(3)$ of 2-norbornen-5-one is, according to the present work, 43:57 $( \pm 2)$ if only the products detectable by GLC are taken into account; 53:47 $( \pm 2)$ if the estimated amount of cis-3-hydroxycyclopentaneacetic acid (5) is also included; and $63: 37( \pm 1)$ if the roughly estimated amount of trans-hydroxycyclopentaneacetic acids (5 and 6 ) is additionally included. The kinetically obtained ratio, 38:62 ( \pm 1 ; see above), agrees best with the first of these values, but the resisting effect of a methyl group at $\mathrm{C}(3)$ with respect to the transfer of the positive charge from $\mathrm{C}(3)$ to the carbonyl 


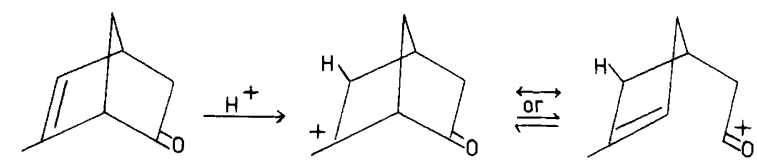

group (eqn. 8) may make the kinetic ratio too small. The other two product-analytic ratios are thus probably more correct. They show that protonation at $\mathrm{C}(2)$ is slightly more favored than at $\mathrm{C}(3)$, which is in agreement with the calculated gas-phase energies of 6- and 5-oxo-2-norbornyl cations $\left(I_{2}\right.$ and $I_{1}$, respectively). ${ }^{6}$ The discrepancy between the kinetic and product-analytic ratios is, however, rather small.

\section{References}

1. Lajunen, M. and Sura, T. Tetrahedron 34 (1978) 189.

2. Krieger, H. Ann. Acad. Sci. Fenn. A 109 (1961).

3. Carrupt, P.-A. and Vogel, P. Tetrahedron Lett. 23 (1982) 2563.

4. Lajunen, M. and Hiukka, R. Acta Chem. Scand., Ser. A39 (1985) 109.

5. Fischer, W., Grob, C. A. and von Sprecher, G. Tetrahedron Lett. (1979) 473; Fischer, W., Grob, C. A., von Sprecher, G. and Waldner, A. Ibid. 1901 and 1905.

6. Carrupt, P.-A. and Vogel, P. J. Phys. Org. Chem. 1 (1988) 287.

7. Freeman, P. K., Balls, D. M. and Brown, D. J. J. Org. Chem. 33 (1968) 2211
8. Masar, S.-E. and Krieger, H. Suom. Kemistil. B 42 (1969) 1.

9. Lippmaa, E., Pehk, T., Paasivirta, J., Belikova, N. and Plate, A. Org. Magn. Reson. 2 (1970) 581.

10. Suginome, H. and Yamada, S. Bull. Chem. Soc. Jpn. 58 (1985) 3055.

11. Hall, H. K., Jr. J. Org. Chem. 28 (1963) 2027; Hill, R. K. and Edwards, A. G. Tetrahedron 21 (1965) 1501.

12. Corey, E. J. and Snider, B. B. J. Org. Chem. 39 (1974) 256

13. Newton, R.F., Reynolds, D. P., Crossland, N.M., Kelly, D. R. and Roberts, S. M. J. Chem. Soc., Perkin Trans. I (1980) 1583.

14. Das Gupta, T.K., Felix, D., Kempe, U.M. and Eschenmoser, A. Helv. Chim. Acta 55 (1972) 2198.

15. Paasivirta, J. Tetrahedron Lett. (1968) 2867; Suom. Kemistil. B 41 (1968) 335; Gassman, P. G. and Zalar, F. V. Tetrahedron Lett. (1964) 3031.

16. Schmid, G. H. and Fitzgerald, P. H. Can. J. Chem. 46 (1968) 3758.

17. Wheeler, O.H. and Granell de Rodriques, E. E. J. Org. Chem. 29 (1964) 1227.

18. Takano, S., Iwata, H. and Ogasawara, K. Heterocycles 12 (1979) 699; Chem. Abstr. 91 (1979) 56723j.

19. Fischer, W., Grob. C. A., von Sprecher, G. and Waldner, A. Helv. Chim. Acta 63 (1980) 816

20. Lajunen, M. and Laaksonen, R. Acta Chem. Scand., Ser. A42 (1988) 254

21. Davis, M. A. J. Org. Chem. 32 (1967) 1161.

22. Lajunen, M. Acc. Chem. Res. 18 (1985) 254.

Received December 28, 1988. 